\title{
Generalized Dyck tilings (Extended Abstract)
}

\author{
Matthieu Josuat-Vergés 1" and Jang Soo Kim थ^" \\ ${ }^{1}$ IGM, Université de Marne-la-Vallée, France \\ ${ }^{2}$ Department of Mathematics, Sungkyunkwan University, Suwon 440-746, South Korea.
}

\begin{abstract}
Recently, Kenyon and Wilson introduced Dyck tilings, which are certain tilings of the region between two Dyck paths. The enumeration of Dyck tilings is related with hook formulas for forests and the combinatorics of Hermite polynomials. The first goal of this work is to give an alternative point of view on Dyck tilings by making use of the weak order and the Bruhat order on permutations. Then we introduce two natural generalizations: $k$-Dyck tilings and symmetric Dyck tilings. We are led to consider Stirling permutations, and define an analogue of the Bruhat order on them. We show that certain families of $k$-Dyck tilings are in bijection with intervals in this order. We enumerate symmetric Dyck tilings and show that certain families of symmetric Dyck tilings are in bijection with intervals in the weak order on signed permutations.

Résumé. Récemment, Kenyon et Wilson ont introduit les pavages de Dyck, qui sont des pavages de la région comprise entre deux chemins de Dyck. L'énumération des pavages de Dyck est reliée aux formules d'équerre sur les forêts et à la combinatoire des polynômes de Hermite. Le premier but de ce travail est de donner un point de vue alternatif sur les pavages de Dyck, en utilisant l'ordre faible et l'ordre de Bruhat sur les permutations. Nous introduisons ensuite deux généralisations naturelles: les $k$-pavages de Dyck et les pavages de Dyck symétriques. Nous sommes amenés à considérer les permutations de Stirling, et définissons un analogue de l'ordre de Bruhat. Nous montrons que certaines familles de $k$-pavages de Dyck sont en bijection avec des intervalles de cet ordre. Nous énumérons les pavages de Dyck symétriques et montrons que certaines familles de pavages de Dyck symétriques sont en bijection avec des intervalles de l'ordre faible sur les permutations signées.
\end{abstract}

Keywords: Dyck tilings, Bruhat order, weak order, Stirling permutations, forests, matchings

\section{Introduction}

Dyck tilings were recently introduced by Kenyon and Wilson [9] in the study of probabilities of statistical physics model called "double-dimer model", and independently by Shigechi and Zinn-Justin [13] in the study of Kazhdan-Lusztig polynomials. Dyck tilings also have connection with fully packed loop configurations [6] and the representation of symmetric groups [5].

The main purpose of this paper is to give a new point of view on Dyck tilings in terms of the weak order and the Bruhat order on permutations and to consider two natural generalizations of Dyck tilings.

\footnotetext{
*Email: matthieu.josuat-verges@univ-mlv.fr

$\dagger^{\dagger}$ Email: jangsookimeskku.edu. The second author was partially supported by Basic Science Research Program through the National Research Foundation of Korea (NRF) funded by the Ministry of Education (NRF-2013R1A1A2061006).
} 


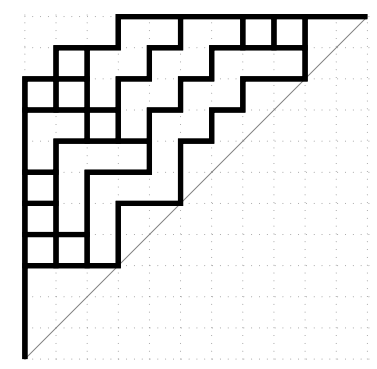

Fig. 1: An example of Dyck tiling.

A Dyck path of length $2 n$ is a lattice path consisting of up steps $(0,1)$ and down steps $(1,0)$ from the origin $(0,0)$ to the point $(n, n)$ which never goes below the line $y=x$. We will also consider a Dyck path $\lambda$ of length $2 n$ as the Young diagram whose border is determined by $\lambda$ and the lines $x=0$ and $y=n$.

Suppose that $\lambda$ and $\mu$ are Dyck paths of length $2 n$ with $\mu$ weakly above $\lambda$. A Dyck tile is a ribbon whose northwest boundary is a Dyck path. A Dyck tiling of $\lambda / \mu$ is a tiling $D$ of the region between $\lambda$ and $\mu$ with Dyck tiles satisfying the cover-inclusive property: if $\eta$ is a tile of $D$, then the translation of $\eta$ by $(1,-1)$ is either completely below $\lambda$ or contained in another tile of $D$. See Figure 1 for an example. We denote by $\mathcal{D}(\lambda / \mu)$ the set of Dyck tilings of $\lambda / \mu$. For $D \in \mathcal{D}(\lambda / \mu)$ we call $\lambda$ and $\mu$ the lower path and the upper path of $D$, respectively. Then the set of Dyck tilings with fixed upper path $\lambda$ is denoted by $\mathcal{D}(\lambda / *)$ and similarly, the set of Dyck tilings with fixed lower path $\mu$ is denoted by $\mathcal{D}(* / \mu)$.

For $D \in \mathcal{D}(\lambda / \mu)$ we have two natural statistics: the area area $(D)$ of the region $\lambda / \mu$ and the number tiles $(D)$ of tiles of $D$. We also consider the statistic $\operatorname{art}(D)=(\operatorname{area}(D)+\operatorname{tiles}(D)) / 2$.

Kenyon and Wilson [9] conjectured the following two formulas:

$$
\begin{aligned}
\sum_{D \in \mathcal{D}(\lambda / *)} q^{\operatorname{art}(D)} & =\frac{[n]_{q} !}{\prod_{x \in F}\left[h_{x}\right]_{q}}, \\
\sum_{D \in \mathcal{D}(* / \mu)} q^{\operatorname{tiles}(D)} & =\prod_{u \in \operatorname{UP}(\mu)}[\operatorname{ht}(u)]_{q},
\end{aligned}
$$

where $F$ is the plane forest corresponding to $\lambda$ and, for a vertex $x \in F, h_{x}$ denotes the hook length of $x$. The set of up steps of a Dyck path $\mu$ is denoted by $\operatorname{UP}(\mu)$ and for $u \in \operatorname{UP}(\mu)$, ht $(u)$ is the number of squares between $u$ and the line $y=x$ plus 1 . Here we use the standard notation for $q$-factorials: $[n]_{q}=1+q+q^{2}+\cdots+q^{n-1}$ and $[n]_{q} !=[1]_{q}[2]_{q} \ldots[n]_{q}$.

Formula (1] was first proved by Kim [10] non-bijectively and then by Kim, Mészáros, Panova, and Wilson [11] bijectively. In [11], they find a bijection between $\mathcal{D}(\lambda / *)$ and increasing labelings of the plane forest corresponding to $\lambda$. Kim [10] and Konvalinka independently proved (2) by finding a bijection between $\mathcal{D}(* / \mu)$ and certain labelings of $\mu$ called Hermite histories.

In this paper we first show that, using the results of Björner and Wachs [2, 3], (1) can be interpreted as the length generating function for permutations $\pi \geq_{L} \sigma$ in the left weak order for a 213-avoiding permutation $\sigma$. We also show that (2) is the length generating function for permutations $\pi \geq \sigma$ in the Bruhat order for a 132-avoiding permutation $\sigma$. We then consider two natural generalizations of Dyck tilings, namely, $k$-Dyck tilings and symmetric Dyck tilings. 


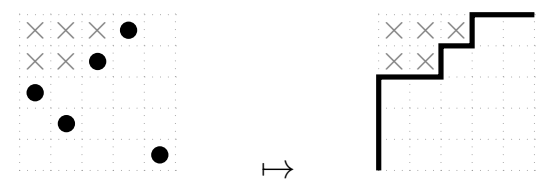

Fig. 2: The bijection from 132-avoiding permutations to Dyck paths. The crosses represent inversions of the permutation 34215 .

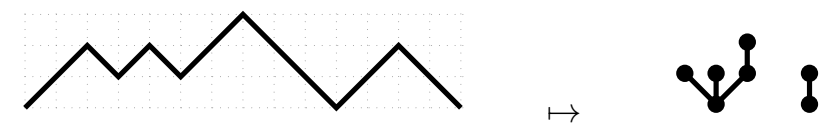

Fig. 3: The bijection from Dyck paths to plane forests. Here the Dyck path is rotated clockwise by an angle of $45^{\circ}$. A pair of matching up step and down step corresponds to a vertex.

The first generalization is $k$-Dyck tiling, where we use $k$-Dyck paths and $k$-Dyck tiles with the same cover-inclusive property. We generalize (2) by finding a bijection between $k$-Dyck tilings and $k$-Hermite histories. We consider $k$-Stirling permutations introduced by Gessel and Stanley [7]. We define a $k$ Bruhat order on $k$-Stirling permutations and show that $k$-Dyck tilings with fixed upper path are in bijection with an interval in this order. We also consider a connection with $k$-regular noncrossing partitions. We generalize (1) to $k$-Dyck tilings with fixed lower path $\lambda$ when $\lambda$ is a zigzag path.

The second generalization is symmetric Dyck tiling, which is invariant under the reflection along a line. We show that symmetric Dyck tilings are in bijection with symmetric matchings and "marked" increasing labelings of symmetric forests. In a special case we show that symmetric Dyck tilings with fixed lower path correspond to an interval of the left weak order on signed permutations.

\section{Dyck tilings as intervals of the Bruhat order and weak order}

As we have seen in the introduction, the two natural points of view for enumerating Dyck tilings are when we fix the upper path, and when we fix the lower path. We show in this section that both can be interpreted in terms of permutations, using respectively the Bruhat order and the (left) weak order, see [1].

We begin by the case of a fixed upper path, and the Bruhat order. We need a bijection $\alpha$ between 132 -avoiding permutations and Dyck paths. This simple bijection is illustrated in Figure 2

We denote by $\mathfrak{S}_{n}$ the set of permutations of $[n]:=\{1,2, \ldots, n\}$. An inversion of $\pi \in \mathfrak{S}_{n}$ is a pair $(i, j)$ of integers $1 \leq i<j \leq n$ such that $\pi(i)>\pi(j)$. The number of inversions of $\pi$ is denoted by $\operatorname{inv}(\pi)$. For a permutation $\tau$, the set of $\tau$-avoiding permutations in $\mathfrak{S}_{n}$ is denoted by $\mathfrak{S}_{n}(\tau)$.

Proposition 2.1 Suppose that a Dyck path $\mu$ corresponds to $\sigma \in \mathfrak{S}_{n}(132)$, then

$$
\sum_{D \in \mathcal{D}(* / \mu)} q^{\mathrm{tiles}(D)}=\sum_{\pi \geq \sigma} q^{\operatorname{inv}(\pi)-\operatorname{inv}(\sigma)}
$$

where $\pi \geq \sigma$ is the Bruhat order on $\mathfrak{S}_{n}$.

Let us turn to the case of a fixed lower path in Dyck tilings. We can identify a Dyck path $\lambda$ with a plane forest as shown in Figure 3 For an increasing labeling $L$ of a plane forest $F$ let post $(L)$ denote 

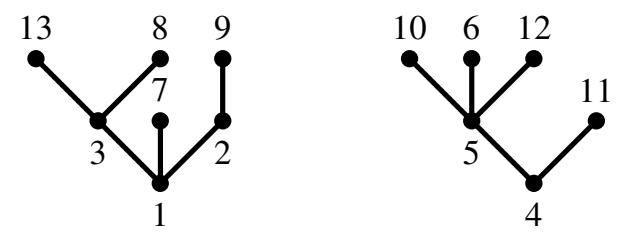

Fig. 4: An increasing labeling $L$ of a forest of size 13 . We have $\operatorname{post}(L)=11,12,6,10,5,4,9,2,7,8,13,3,1$.

the permutation obtained by reading $L$ from right to left using post-order. See Figure 4 . It is easy to see that there is a unique increasing labeling $L_{0}$ of $F$ such that $\operatorname{inv}\left(\operatorname{post}\left(L_{0}\right)\right)$ is minimal. In this case the permutation $\pi_{0}=\operatorname{post}\left(L_{0}\right)$ is 213 -avoiding.

Proposition 2.2 Let $\lambda$ be a Dyck path with corresponding plane forest $F$. Let $L_{0}$ be the unique increasing labeling $L_{0}$ of $F$ such that $\operatorname{inv}\left(\operatorname{post}\left(L_{0}\right)\right)$ is minimal, and $\pi_{0}=\operatorname{post}\left(L_{0}\right)$. Then

$$
\sum_{D \in \mathcal{D}(\lambda / *)} q^{\operatorname{art}(D)}=\sum_{\pi \geq_{L} \pi_{0}} q^{\operatorname{inv}(\pi)-\operatorname{inv}\left(\pi_{0}\right)},
$$

where $\geq_{L}$ is the left weak order on $\mathfrak{S}_{n}$.

Note that the inversion generating function of increasing labelings of a plane forest is given by a hook length formula [2]. The fact that some intervals for the weak order have a generating function given by a hook length formula follows from [3].

\section{$3 k$-Dyck tilings}

A $k$-Dyck path is a lattice path consisting of up steps $(0,1)$ and down steps $(1,0)$ from the origin $(0,0)$ to the point $(k n, n)$ which never goes below the line $y=x / k$. Let $\operatorname{Dyck}^{(k)}(n)$ denote the set of $k$-Dyck paths from $(0,0)$ to $(k n, n)$.

A $k$-Dyck tile is a ribbon in which the centers of the cell form a $k$-Dyck path. A (cover-inclusive) $k$-Dyck tiling is a tiling $D$ of the region $\lambda / \mu$ between $\lambda, \mu \in \operatorname{Dyck}^{(k)}(n)$ with $k$-Dyck tiles satisfying the cover-inclusive property: if $\eta$ is a tile in $D$, then the translation of $\eta$ by $(1,-1)$ is either completely below $\lambda$ or contained in another tile of $D$. See Figure 5 for an example. We denote by $\mathcal{D}^{(k)}(\lambda / \mu)$ the set of $k$-Dyck tilings of $\lambda / \mu$. We also denote by $\mathcal{D}^{(k)}(\lambda / *)$ and $\mathcal{D}^{(k)}(* / \mu)$ the sets of $k$-Dyck tilings with fixed lower path $\lambda$ and with fixed upper path $\mu$, respectively.

For an up step $u$ of $\mu \in \operatorname{Dyck}^{(k)}(n)$, we define ht $(u)$ to be the number of squares between $u$ and the line $y=x / k$ plus 1 . For $D \in \mathcal{D}^{(k)}(\lambda / \mu)$, there are two natural statistics: the number tiles $(D)$ of tiles in $D$ and the area area $(D)$ of the region occupied by $D$. We also define

$$
\operatorname{art}_{k}(D)=\frac{k \cdot \operatorname{area}(D)+\operatorname{tiles}(D)}{k+1} .
$$

The following theorem is a generalization of (2).

Theorem 3.1 For $\mu \in \operatorname{Dyck}^{(k)}(n)$, we have

$$
\sum_{D \in \mathcal{D}^{(k)}(* / \mu)} q^{\mathrm{tiles}(D)}=\prod_{u \in \mathrm{UP}(\mu)}[\mathrm{ht}(u)]_{q} .
$$




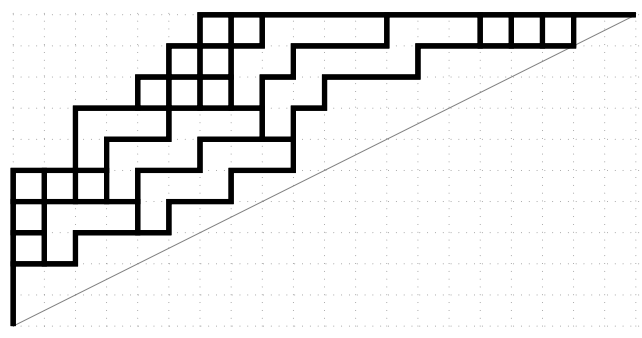

Fig. 5: An example of 2-Dyck tiling.

For an arbitrary $\lambda \in \operatorname{Dyck}^{(k)}(n)$, there does not seem to be a product formula for the cardinality of $\mathcal{D}^{(k)}(\lambda / *)$. However, when $\lambda$ is a zigzag path there is a nice generalization of (1).

Theorem 3.2 Let $\lambda$ be the path $u^{n_{1}} d^{k n_{1}} u^{n_{2}} d^{k n_{2}} \cdots u^{n_{\ell}} d^{k n_{\ell}}$, where $u$ means an up step and $d$ means a down step. Then we have

$$
\sum_{D \in \mathcal{D}^{(k)}(\lambda / *)} q^{\operatorname{art}_{k}(D)}=\left[\begin{array}{c}
k n_{1}+n_{2} \\
n_{2}
\end{array}\right]_{q}\left[\begin{array}{c}
k\left(n_{1}+n_{2}\right)+n_{3} \\
n_{3}
\end{array}\right]_{q} \ldots\left[\begin{array}{c}
k\left(n_{1}+\cdots+n_{\ell-1}\right)+n_{\ell} \\
n_{\ell}
\end{array}\right]_{q},
$$

where $\left[\begin{array}{l}a \\ b\end{array}\right]_{q}=\frac{[a]_{q} !}{[b-a]_{q} ![b]_{q} !}$.

\section{$4 \quad k$-Stirling permutations and the $k$-Bruhat order}

In this section we consider $k$-Stirling permutations which were introduced by Gessel and Stanley [7] for $k=2$ and studied further for general $k$ by Park [12].

A $k$-Stirling permutation of size $n$ is a permutation of the multiset $\left\{1^{k}, 2^{k}, \ldots, n^{k}\right\}$ such that if an integer $j$ appears between two $i$ 's then $i>j$. Let $\mathfrak{S}_{n}^{(k)}$ denote the set of $k$-Stirling permutations of size $n$. We can represent a $k$-Stirling permutation $\pi=\pi_{1} \pi_{2} \ldots \pi_{k n}$ as the $n \times k n$ matrix $M=\left(M_{i, j}\right)$ defined by $M_{i, j}=1$ if $\pi_{j}=i$ and and $M_{i, j}=0$ otherwise. Then the entries of $M$ are 0 's and 1's such that each column contains exactly one 1 , each row contains $k 1$ 's, and it does not contain the following matrix as a submatrix:

$$
\left(\begin{array}{lll}
1 & 0 & 1 \\
0 & 1 & 0
\end{array}\right)
$$

A $k$-inversion of $\pi \in \mathfrak{S}_{n}^{(k)}$ is a pair $(i, j) \in[n] \times[k n]$ such that $\pi_{j}>i$ and the first $i$ appears after $\pi_{j}$. Equivalently we can think of a $k$-inversion as an entry in the matrix of $\pi$ which has $k 1$ 's to the left in the same row and one 1 below in the same column. We denote the set of $k$-inversions of $\pi$ by $\operatorname{INV}_{k}(\pi)$, and $\operatorname{inv}_{k}(\pi)=\left|\operatorname{INV}_{k}(\pi)\right|$. It is easy to see that $\pi$ is determined by its $k$-inversions.

Definition 1 We define the $k$-Bruhat order on $\mathfrak{S}_{n}^{(k)}$ given by the cover relation $\sigma \lessdot \pi$ if $\pi$ is obtained from $\sigma$ by exchanging the two numbers in positions $a_{1}$ and $a_{k+1}$ for some integers $a_{1}<a_{2}<\cdots<a_{k+1}$ satisfying the following conditions:

$$
\text { 1. } \sigma_{a_{1}}=\sigma_{a_{2}}=\cdots=\sigma_{a_{k}}<\sigma_{a_{k+1}} \text {, and }
$$




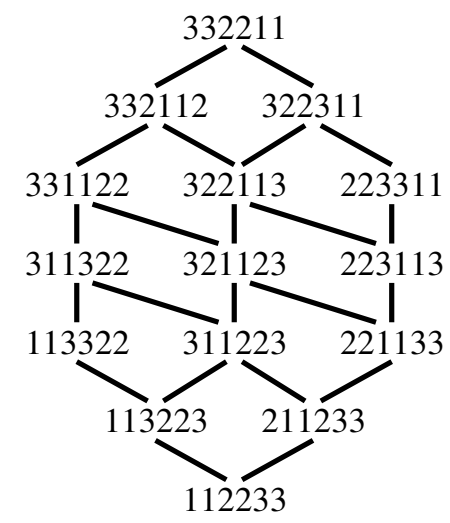

Fig. 6: The Hasse diagram of $\mathfrak{S}_{3}^{(2)}$.

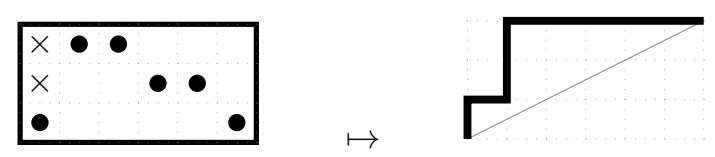

Fig. 7: The bijection $\alpha$ in Proposition 4.3

2. for all $a_{k}<i<a_{k+1}$ we have either $\sigma_{i}<\sigma_{a_{k}}$ or $\sigma_{i}>\sigma_{a_{k+1}}$.

Note that the 1-Bruhat order is the usual Bruhat order. Figure 6 illustrates the $k$-Bruhat order.

Remark 1 The elements of $\mathfrak{S}_{n}^{(k)}$ where all occurrences of $i$ are consecutive for any $i$, form a subset in natural bijection with permutations. In general $(k>1$ and $n>2)$, the induced order on this subset is strictly contained in the Bruhat order, and contains strictly the left weak order.

Proposition 4.1 The $k$-Bruhat order gives a graded poset $\mathfrak{S}_{n}^{(k)}$ with rank function $\operatorname{inv}_{k}$.

Proposition 4.2 Suppose that $\sigma \in \mathfrak{S}_{n}^{(k)}$ is 132-avoiding. Then, for $\pi \in \mathfrak{S}_{n}^{(k)}$, we have $\sigma \leq \pi$ if and only if $\operatorname{INV}_{k}(\sigma) \subseteq \operatorname{INV}_{k}(\pi)$.

There is a trivial bijection between $k$-Dyck tilings without tiles, and $k$-Dyck paths. As for the $k$-Stirling permutations, we have:

Proposition 4.3 The inversions of a 132-avoiding $k$-Stirling permutation $\sigma$ are arranged as the cells of a top-left justified Ferrers diagram. Define a path $\alpha(\sigma)$ from the bottom left to the top right corner, and following the boundary of this Ferrers diagram with up and right steps. Then $\alpha$ is a bijection between 132-avoiding $k$-Stirling permutations and $k$-Dyck paths of length $n$, in particular both are counted by the Fuss-Catalan numbers $\frac{1}{k n+1}\left(\begin{array}{c}(k+1) n \\ n\end{array}\right)$.

The proof is simple and similar to the case $k=1$. For example, see Figure 7

We now have a generalization of Proposition 2.1. 
Theorem 4.4 If $\mu$ is a k-Dyck path corresponding to $\sigma \in \mathfrak{S}_{n}^{(k)}(132)$, then

$$
\sum_{D \in \mathcal{D}_{n}^{(k)}(* / \mu)} q^{\mathrm{tiles}(D)}=\sum_{\pi \geq \sigma} q^{\operatorname{inv}_{k}(\pi)-\operatorname{inv}_{k}(\sigma)} .
$$

\section{$5 \quad k$-regular noncrossing partitions}

In this section, we take another point of view on the $k$-Stirling permutations studied in the previous section.

Definition 2 We denote by $\mathrm{NC}_{n}^{(k)}$ the set of $k$-regular noncrossing partitions of size n, i.e. set partitions of $[k n]$ such that each block contains $k$ elements, and there are no integers $a<b<c<d$ such that $a, c$ are in one block, and $b, d$ in another block. To each $k$-regular noncrossing partition $\pi$ of $[k n]$, we define its nesting poset $\operatorname{Nest}(\pi)$ as follows: the elements of the poset are the blocks of $\pi$, and $x \leq y$ in the poset when the block $x$ lies between two elements of the block $y$.

There is a natural way to consider a $k$-Stirling permutation as a linear extension of the nesting poset of a $k$-regular noncrossing partition.

Proposition 5.1 There is a bijection between $\mathfrak{S}_{n}^{(k)}$ and the pairs $(\pi, E)$ where $\pi \in \mathrm{NC}_{n}^{(k)}$ and $E$ is a linear extension of the poset $\operatorname{Nest}(\pi)$.

Note that since the nesting order of a $k$-noncrossing partition is a forest, there is a hook length formula for the number of linear extensions [3]. If $x \in \operatorname{Nest}(\pi)$, let $h_{x}$ denote the number of elements above $x$ in the nesting poset, then the number of linear extensions of $\pi \in \mathrm{NC}_{n}^{(k)}$ is

$$
\frac{n !}{\prod_{x \in \operatorname{Nest}(\pi)} h_{x}} .
$$

This gives the following formula for the number of $k$-Stirling permutations.

Proposition 5.2 (Multifactorial hook length formula)

$$
1(k+1)(2 k+1) \ldots((n-1) k+1)=\sum_{\pi \in \mathrm{NC}_{n}^{(k)}} \frac{n !}{\prod_{x \in \operatorname{Nest}(\pi)} h_{x}}
$$

In the case $k>2$, it is not clear how to follow the $q$-statistic, but for $k=2$ we can use the bijection between noncrossing matchings and plane forests (which is just $\pi \mapsto \operatorname{Nest}(\pi)$ ), and use the $q$-hook length formula from [2]. We get a hook length formula for $[1]_{q}[3]_{q} \ldots[2 n-1]_{q}$.

Proposition 5.3 ( $q$-double factorial hook length formula) We have

$$
[1]_{q}[3]_{q} \ldots[2 n-1]_{q}=\sum_{F}[n]_{q^{2}} ! \prod_{v \in F} \frac{q^{h_{v}-1}}{\left[h_{v}\right]_{q^{2}}},
$$

where the sum is over all plane forests $F$ with $n$ vertices. 

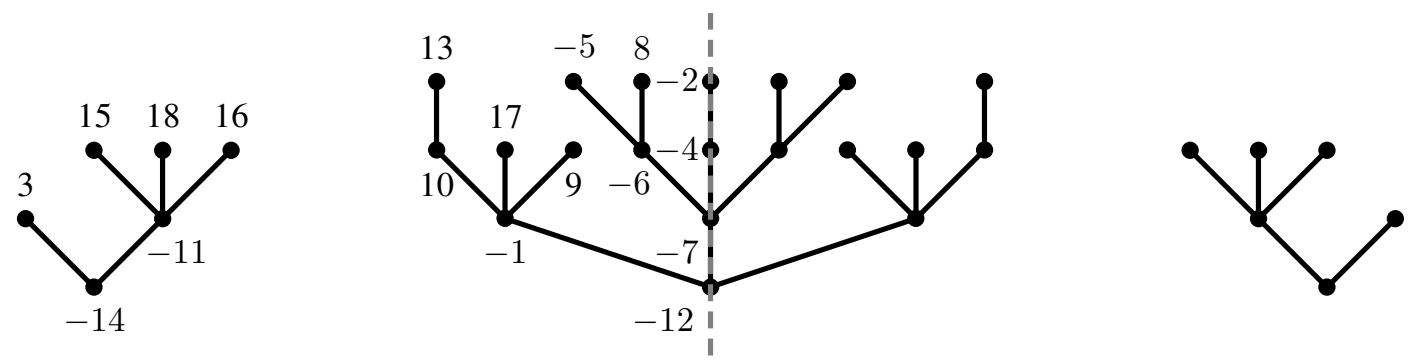

Fig. 8: A symmetric plane tree of size 18 and a type $B$ increasing labeling $L$ of it. The center line is the dashed line. We have $\operatorname{post}(L)=-2,-4,8,-5,-6,-7,9,17,13,10,-1,-12,16,18,15,-11,3,-14$.

\section{Type $B$ increasing forests and the weak order on $B_{n}$}

Let $[n]=\{1,2, \ldots, n\}$ and $[ \pm n]=\{1,2, \ldots, n,-1,-2, \ldots,-n\}$. A signed permutation is a permutation $\pi$ of $[ \pm n]$ such that $\pi(-i)=-\pi(i)$. We can represent $\pi$ as the sequence $\pi_{1} \pi_{2} \ldots \pi_{n}$ where $\pi_{i}=\pi(i)$. Let $B_{n}$ denote the set of signed permutations of $[ \pm n]$. We refer the reader to [1] for basic definitions and properties of $B_{n}$ such as the length function $\ell_{B}$, the Bruhat order and the weak order.

For simplicity we will write a negative integer $-i$ as $\bar{i}$ to express a signed permutation.

A symmetric plane forest is a plane forest which is invariant under the reflection about a line, called the center line. A center vertex is a vertex on the center line. The (weakly) left part of a symmetric plane forest is the subforest consisting of vertices on or to the left of the center line. The size of a symmetric plane forest is the number of vertices in the left part of it.

Let $F$ be a symmetric plane forest of size $n$. A type $B$ increasing labeling of $F$ is a labeling of the left part of $F$ with $[ \pm n]$ such that

1. the labels are increasing from roots to leaves,

2. for each $i \in[n]$, exactly one of $i$ or $-i$ appears,

3. the label of every center vertex is negative.

See Figure 8 for an example of type $B$ increasing labeling. We denote by the set of type $B$ increasing labelings of $F$ by $\operatorname{INC}_{B}(F)$.

For $L \in \mathrm{INC}_{B}(F)$ we define post $(L)$ to be the signed permutation obtained by reading $F$ from right to left using post-order. It is easy to see that there is a unique type $B$ increasing labeling $L_{0} \in \operatorname{INC}_{B}(F)$ such that $\ell_{B}\left(\operatorname{post}\left(L_{0}\right)\right)$ is minimal.

For $\pi=\pi_{1} \pi_{2} \ldots \pi_{n} \in B_{n}$ we define $\operatorname{NEG}(\pi)=\left\{i: \pi_{i}<0\right\}$,

$$
\operatorname{INV}(\pi)=\left\{(i, j): 1 \leq i<j \leq n, \pi_{i}>\pi_{j}\right\}
$$

and $\operatorname{NEGPAR}(\pi)$ to be the integer partition with part $\left|\pi_{i}\right|$ for each $\pi_{i}<0$. We will consider $\operatorname{NEGPAR}(\pi)$ as the set of the cells in the Young diagram.

The following proposition is well known, for instance, see [1, Proposition 8.1.1].

Proposition 6.1 For $\pi \in B_{n}$ we have $\ell_{B}(\pi)=|\operatorname{INV}(\pi)|+|\operatorname{NEGPAR}(\pi)|$. 


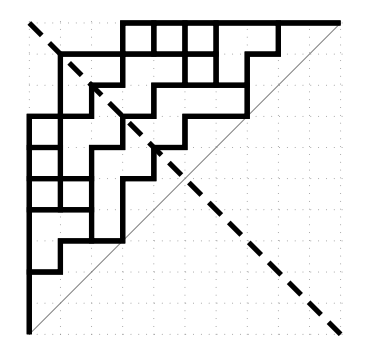

Fig. 9: An example of symmetric Dyck tiling. It is symmetric about the dashed line.

We have a simple criterion to determine whether two signed permutations are comparable in the left weak order $\leq_{L}$.

Proposition 6.2 For $\sigma, \pi \in B_{n}$, we have $\sigma \leq_{L} \pi$ if and only if $\operatorname{INV}(\sigma) \subseteq \operatorname{INV}(\pi)$ and $\operatorname{NEGPAR}(\sigma) \subseteq$ $\operatorname{NEGPAR}(\pi)$. Moreover, in this case we have $\operatorname{NEG}(\sigma) \subseteq \operatorname{NEG}(\pi)$.

Proposition 6.3 Let $F$ be a symmetric plane forest of size $n$. Let $L_{0}$ be the unique type $B$ increasing labeling of $F$ with $\ell_{B}\left(\operatorname{post}\left(L_{0}\right)\right)$ minimal. Then

$$
\left\{\operatorname{post}(L): L \in \operatorname{INC}_{B}(F)\right\}=\left[\operatorname{post}\left(L_{0}\right), \overline{1} \overline{2} \ldots \bar{n}\right],
$$

where $[\sigma, \pi]$ means the interval $\left\{\tau \in B_{n}: \sigma \leq_{L} \tau \leq_{L} \pi\right\}$ in the poset $\left(B_{n}, \leq_{L}\right)$.

\section{Symmetric Dyck tilings and marked increasing forests}

Let $F$ be a symmetric plane forest of size $n$. A marked increasing labeling of $F$ is a labeling of the left part of $F$ with $[n]$ such that the labels are increasing from roots to leaves, each integer appears exactly once, and each non-center vertex may be marked with $*$. We denote the set of marked increasing labelings of $F$ by $\operatorname{INC}^{*}(F)$. Note that in general $\operatorname{INC}^{*}(F)$ and $\operatorname{INC}_{B}(F)$ have different cardinalities.

Let $L \in \mathrm{INC}^{*}(F)$. We denote by $\operatorname{MARK}(L)$ the set of labels of the marked vertices in $L$. An inversion of $L$ is a pair of vertices $(u, v)$ such that $L(u)>L(v), u$ and $v$ are incomparable, and $u$ is to the left of $v$. Let $\operatorname{INV}(L)$ denote the set of inversions of $L$.

A Dyck path $\lambda$ of length $2 n$ is called symmetric if it is invariant under the reflection along the line $x+y=n$. For two symmetric Dyck paths $\lambda$ and $\mu$ of length $2 n$, a Dyck tiling of $\lambda / \mu$ is called symmetric if it is invariant under the reflection along the line $x+y=n$. See Figure 9 for an example of symmetric Dyck tiling. We denote by $\mathcal{D}_{\text {sym }}(\lambda / \mu)$ the set of symmetric Dyck tilings of shape $\lambda / \mu$.

For a symmetric Dyck tiling $D$, a positive tile is a tile which lies strictly to the left of the center line, and a zero tile is a tile which intersects with the center line. We denote by tiles $+(D)$ and tiles ${ }_{0}(D)$ the number of positive tiles and zero tiles, respectively. Note that the total number of tiles in $D$ is tiles $(D)=$ $2 \cdot \operatorname{tiles}_{+}(D)+$ tiles $_{0}(D)$. We also define area $+(D)$ and $\operatorname{area}_{0}(D)$ to be the total area of positive tiles and zero tiles in $D$, respectively, and

$$
\operatorname{art}_{+}(D)=\frac{\operatorname{area}_{+}(D)+\operatorname{tiles}_{+}(D)}{2}, \quad \operatorname{art}_{0}(D)=\frac{\operatorname{area}_{0}(D)+\operatorname{tiles}_{0}(D)}{2} .
$$

For example, if $D$ is the symmetric Dyck tiling in Figure 9, we have tiles $+(D)=5$, tiles $_{0}(D)=3$, $\operatorname{area}_{+}(D)=7, \operatorname{area}_{0}(D)=19, \operatorname{art}_{+}(D)=(7+5) / 2=6$, and $\operatorname{art}_{0}(D)=(19+3) / 2=11$. 
Theorem 7.1 Let $F$ be a symmetric plane forest of size $n$ and $\lambda$ the corresponding Dyck path. Then there is a bijection $\phi: \operatorname{INC}^{*}(F) \rightarrow \mathcal{D}_{\mathrm{sym}}(\lambda / *)$ such that if $\phi(L)=D$, then $\operatorname{tiles}_{0}(D)=|\operatorname{MARK}(L)|$ and

$$
\operatorname{art}_{+}(D)+\operatorname{art}_{0}(D)=|\operatorname{INV}(L)|+\sum_{i \in \operatorname{MARK}(L)}(n+1-i) .
$$

Corollary 7.2 Let $F$ be a symmetric plane forest of size $n$ with $k$ center vertices and $\lambda$ the corresponding Dyck path. Then

$$
\left|\mathcal{D}_{\text {sym }}(\lambda / *)\right|=2^{n-k} \frac{n !}{\prod_{x \in T} h_{x}} .
$$

If $k=0$, we have

$$
\sum_{D \in \mathcal{D}_{\text {sym }}(\lambda / *)} q^{\operatorname{art}_{+}(D)+\operatorname{art}_{0}(D)} t^{\mathrm{tiles}_{0}(D)}=(1+t q)\left(1+t q^{2}\right) \cdots\left(1+t q^{n}\right) \frac{[n]_{q} !}{\prod_{x \in T}\left[h_{x}\right]_{q}} .
$$

As is already mentioned $\operatorname{INC}_{B}(F)$ and $\operatorname{INC}^{*}(F)$ have different cardinalities. However, if $F$ has no center vertice, then there is a simple bijection between $\operatorname{INC}_{B}(F)$ and $\operatorname{INC}^{*}(F)$.

Corollary 7.3 Let $F$ be a symmetric plane forest of size $n$ with no center vertices and $\lambda$ the corresponding Dyck path. Let $L_{0}$ be the unique type $B$ increasing labeling of $F$ with $\ell_{B}\left(\operatorname{post}\left(L_{0}\right)\right)$ minimal and $\pi_{0}=$ $\operatorname{post}\left(L_{0}\right)$. Then

$$
\sum_{D \in \mathcal{D}_{\mathrm{sym}}(\lambda / *)} q^{\operatorname{art}_{+}(D)+\operatorname{art}_{0}(D)} t^{\mathrm{tiles}_{0}(D)}=\sum_{\pi \geq_{L} \pi_{0}} q^{\ell_{B}(\pi)-\ell_{B}\left(\pi_{0}\right)} t^{|\mathrm{NEG}(\pi)|-\left|\operatorname{NEG}\left(\pi_{0}\right)\right|} .
$$

\section{Symmetric Dyck tilings and symmetric Hermite histories}

For a symmetric Dyck path $\mu$ of length $2 n$, let $\mu^{+}$denote the subpath consisting of the first $n$ steps. Note that each up step is matched with a unique down step in a Dyck path. An up step of $\mu^{+}$is called matched if the corresponding down step in $\mu$ lies in $\mu^{+}$and unmatched otherwise.

A symmetric Hermite history is a symmetric Dyck path $\mu$ with a labeling of the up steps of $\mu^{+}$in such a way that every matched up step of height $h$ has label $i \in\{0,1, \ldots, h-1\}$ and the labels $a_{1}, a_{2}, \ldots, a_{k}$ of the unmatched up steps form an involutive sequence. Here, a sequence is called involutive if it can be obtained by the following inductive way.

- The empty sequence is defined to be involutive.

- The sequence (0) is the only involutive sequence of length 1 .

- For $k \geq 2$, an involutive sequence of length $k$ is either the sequence obtained from an involutive sequence of length $k-1$ by adding 0 at the end or the sequence obtained from an involutive sequence of length $k-2$ by adding an integer $r$ at the end and inserting a 0 before the last $r$ integers, including the newly added integer, for some $1 \leq r \leq k-1$.

From the definition it is clear that the number $v_{k}$ of involutive sequences of length $k$ satisfies the recurrence $v_{k}=v_{k-1}+(k-1) v_{k-2}$ with initial conditions $v_{0}=v_{1}=1$. Thus $v_{k}$ is equal to the number of involutions in $\mathfrak{S}_{n}$.

We denote by $\mathcal{H}_{\text {sym }}(\mu)$ be the set of symmetric Hermite histories on $\mu$. For $H \in \mathcal{H}_{\text {sym }}(\mu)$, let $\|H\|$ be the sum of labels in $H$ and $\operatorname{pos}(H)$ the number of positive labels on unmatched up steps. 
Proposition 8.1 There is a bijection $\psi: \mathcal{H}_{\mathrm{sym}}(\mu) \rightarrow \mathcal{D}_{\mathrm{sym}}(* / \mu)$ such that if $\psi(H)=D$, then $\|H\|=$ tiles $_{+}(D)+\operatorname{tiles}_{0}(D)$ and $\operatorname{pos}(H)=\operatorname{tiles}_{0}(D)$. Thus,

$$
\sum_{D \in \mathcal{D}_{\mathrm{sym}}(* / \mu)} q^{\mathrm{tiles}_{+}(D)+\operatorname{tiles}_{0}(D)} t^{\mathrm{tiles}_{0}(D)}=\sum_{H \in \mathcal{H}_{\mathrm{sym}}(\mu)} q^{\|H\|} t^{\operatorname{pos}(H)}
$$

Corollary 8.2 Let $\mu$ be a symmetric Dyck path such that $\mu^{+}$has $k$ unmatched up steps. Then

$$
\sum_{D \in \mathcal{D}_{\mathrm{sym}}(* / \mu)} q^{\mathrm{tiles}_{+}(D)+\operatorname{tiles}_{0}(D)} t^{\mathrm{tiles}_{0}(D)}=f_{k}(q, t) \prod_{u \in \operatorname{UP}\left(\mu^{+}\right)}[\operatorname{ht}(u)]_{q},
$$

where $\operatorname{UP}\left(\mu^{+}\right)$is the set of matched up steps in $\mu^{+}$and $f_{k}(q, t)$ is defined by $f_{0}(q, t)=f_{1}(q, t)=1$ and $f_{k}(q, t)=f_{k-1}(q, t)+t q[k-1]_{q} f_{k-2}(q, t)$ for $k \geq 2$.

A symmetric matching is a matching on $[ \pm n]$ such that if $\{i, j\}$ is an arc, then $\{-i,-j\}$ is also an arc. We denote by $\mathcal{M}_{\text {sym }}(n)$ the set of symmetric matchings on $[ \pm n]$. Note that symmetric matchings are in bijection with fixed-point-free involutions in $B_{n}$.

Let $M \in \mathcal{M}_{\text {sym }}(n)$. A symmetric crossing of $M$ is a pair of arcs $\{a, b\}$ and $\{c, d\}$ satisfying $a<c<$ $b<d$ and $b, d>0$. A symmetric crossing $(\{a, b\},\{c, d\})$ is called self-symmetric if $\{c, d\}=\{-a,-b\}$. We denote by $\operatorname{cr}(M)$ and $\operatorname{sscr}(M)$ the number of symmetric crossings and self-symmetric crossings of $M$, respectively.

For a symmetric Dyck path $\mu$ of length $2 n$, let $\mathcal{M}_{\text {sym }}(\mu)$ denote the set of symmetric matchings $M$ on $[ \pm n]$ such that the $i$ th smallest vertex of $M$ is a left vertex of an arc if and only if the $i$ th step of $\mu$ is an up step.

Proposition 8.3 We have

$$
\sum_{H \in \mathcal{H}_{\mathrm{sym}}(\mu)} q^{\|H\|_{1} \operatorname{pos}(H)}=\sum_{M \in \mathcal{M}_{\mathrm{sym}}(\mu)} q^{\operatorname{cr}(M)} t^{\mathrm{sscr}(M)} .
$$

For $D \in \mathcal{D}_{\text {sym }}(* / \mu)$, let ht $(D)$ denote the number of unmatched up steps in $\mu^{+}$. Using the result in [4] and [8] on a generating function for partial matchings we obtain the following formula.

Proposition 8.4 We have

$$
\begin{aligned}
& \sum_{D \in \mathcal{D}_{\text {sym }}(n)} q^{\text {tiles }_{+}(D)+\operatorname{tiles}_{0}(D)} t^{\operatorname{tiles}_{0}(D)} s^{\operatorname{ht}(D)}
\end{aligned}
$$

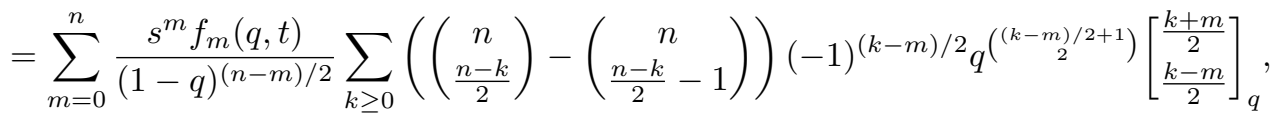

where $f_{m}(q, t)$ is defined in Corollary 8.2

If $t=s=0$ in the above proposition, then we get the generating function for the usual Dyck tilings according to the number of tiles. 


\section{References}

[1] A. Björner and F. Brenti. Combinatorics of Coxeter groups, volume 231 of Graduate Texts in Mathematics. Springer, New York, 2005.

[2] A. Björner and M. L. Wachs. q-hook length formulas for forests. J. Combin. Theory Ser. A, 52(2):165-187, 1989.

[3] A. Björner and M. L. Wachs. Permutation statistics and linear extensions of posets. J. Combin. Theory Ser. A, 58(1):85-114, 1991.

[4] J. Cigler and J. Zeng. A curious $q$-analogue of Hermite polynomials. J. Combin. Theory Ser. A, 118(1):9-26, 2011.

[5] M. Fayers. Dyck tilings and the homogeneous Garnir relations for graded Specht modules. arXiv:1309.6467, 2013.

[6] I. Fischer and P. Nadeau. Fully packed loops in a triangle: matchings, paths and puzzles. arXiv:1209.1262, 2012.

[7] I. Gessel and R. P. Stanley. Stirling polynomials. J. Combinatorial Theory Ser. A, 24(1):24-33, 1978.

[8] M. Josuat-Vergès. Rook placements in Young diagrams and permutation enumeration. Adv. in Appl. Math., 47:1-22, 2011.

[9] R. W. Kenyon and D. B. Wilson. Double-dimer pairings and skew Young diagrams. Electron. J. Combin., 18:\#P130, 2011.

[10] J. S. Kim. Proofs of two conjectures of Kenyon and Wilson on Dyck tilings. J. Combin. Theory Ser. A, 119(8):1692-1710, 2012.

[11] J. S. Kim, K. Mészáros, G. Panova, and D. B. Wilson. Dyck tilings, increasing trees, descents, and inversions. Journal of Combinatorial Theory, Series A, 122:9-27, 2014.

[12] S. Park. The $r$-multipermutations. J. Combin. Theory Ser. A, 67(1):44-71, 1994.

[13] K. Shigechi and P. Zinn-Justin. Path representation of maximal parabolic Kazhdan-Lusztig polynomials. J. Pure Appl. Algebra, 216(11):2533-2548, 2012. 